\title{
Uso de amplificadores sensíveis à fase para medidas de precisão: obtenção da constante de Faraday com campo magnético alternado ou estático
}

Use of phase-sensitive amplifiers for high precision measurements: Faraday constant determination with an alternating or static magnetic field

\author{
Rubens Travassos Augusto Filho ${ }^{1}$, André Luís dos Santos Romero1@, \\ Emerson Cristiano Barbano ${ }^{2}$, Lino Misoguti ${ }^{* 1}$ \\ ${ }^{1}$ Universidade de São Paulo, Instituto de Física de São Carlos, 13560-970, São Carlos, SP, Brasil. \\ ${ }^{2}$ Universidade Federal do Paraná, Departamento de Física, 81531-980, Curitiba, PR, Brasil.
}

Recebido em 28 de maio de 2021. Aceito em 03 de junho de 2021.

\begin{abstract}
Neste trabalho apresentamos alguns dos conceitos fundamentais que, explicam como os amplificadores sensíveis à fase funcionam e, como eles podem ser explorados para projetar métodos experimentais para medidas precisas de grandezas físicas. Mais especificamente, propomos o uso de um amplificador sensível à fase de fase dupla em dois experimentos simples para medida da constante de Verdet nas condições de campos magnéticos alternados e estáticos fracos. Caracterizamos a água e o etanol, bem como misturas dos dois solventes, uma vez que esses líquidos são abundantes, de baixo custo, de fácil manipulação e apresentam constantes de Verdet pequenas, sendo adequados para o teste da sensibilidade e precisão dos experimentos. Também, estudamos as dispersões cromáticas pelas medidas das constantes de Verdet em três comprimentos de onda: $480 \mathrm{~nm}, 540 \mathrm{~nm}$ e, $640 \mathrm{~nm}$. Os bons resultados puderam ser comprovados pela correta concordância entre os dados obtidos com os dois tipos de campo magnético, e também com os valores encontrados na literatura.
\end{abstract}

Palavras-chave: Lock-in, Rotação Faraday, Lei de Malus, Dispersão do índice de refração.

In this work, we present some of the fundamental concepts which explain how lock-in amplifiers work and how they can be explored to design experimental methods for precise physical quantity measurements. More specifically, we propose to use a dual-phase lock-in amplifier in two simple experiments for measuring Verdet constant at weak alternating and static magnetic field conditions. We characterize water and ethanol and mixtures of the two solvents since these liquids are abundant, low cost, easy to handle, and have small Verdet constants, being suitable for testing the sensibility and precision of the experiments. Also, we studied the chromatic dispersions by measuring the Verdet constants in three different wavelengths: $480 \mathrm{~nm}, 540 \mathrm{~nm}$ and, $640 \mathrm{~nm}$. Good results could be confirmed by the fair agreement between the data obtained with the two type of magnetic fields and, also, with values found in the literature.

Keywords: Lock-in, Faraday rotation, Malus Law, Refractive index dispersion.

\section{Introdução}

Os amplificadores sensíveis à fase (ASF) ou amplificadores lock-ins, são equipamentos utilizados rotineiramente em laboratórios de pesquisa para medidas de sinais elétricos com precisão, baixo ruído e com alta sensibilidade. Apesar dos altos custos, são equipamentos essenciais em muitas medidas de precisão e podem ser encontrados em muitos laboratórios de ensino de Física Moderna. Sob o ponto de vista de ensino, é muito importante salientar o princípio de funcionamento desses amplificadores para se poder entender como os experimentos podem ser implementados para obtenção de melhores dados. Neste estudo, em particular, propomos medir a constante de Verdet pelo efeito Faraday. O efeito

\footnotetext{
*Endereço de correspondência: misoguti@ifsc.usp.br
}

Faraday, por sua vez, é a rotação do plano de polarização da luz linearmente polarizada durante a propagação por um meio sujeito a um campo magnético externo paralelo à direção de propagação [1-4].

Por se tratar de uma propriedade importante e fundamental dos materiais, o efeito Faraday é bastante estudado nos laboratórios de Física Moderna [5]. Usualmente, para se determinar a constante de Verdet pelo efeito Faraday, grandes equipamentos com altos campos magnéticos estáticos ( kilogauss ou mais) são aplicados para que ângulos de rotação macroscópicos sejam induzidos e detectados. No entanto, com o uso de técnicas sensíveis à fase, é possível usar um dispositivo muito mais simples e compacto, pois campos magnéticos menores podem ser aplicados, uma vez que ângulos de rotação menores podem ser medidos. Por exemplo, podemos usar um simples solenoide oco com 
duas janelas, para o nosso caso de estudo de líquidos. Neste trabalho, vamos considerar duas maneiras nas quais é possível determinar a constante de Verdet usando técnicas sensíveis à fase, ou seja, com o uso de um ASF, sem a necessidade de aplicação de campos magnéticos muito altos. A primeira (segunda) explora o uso de campos magnéticos alternados (estáticos) obtidos com a aplicação de correntes alternadas (contínuas) num solenoide [6]. Neste segundo caso, por exemplo, é possível determinar pequenas rotações da polarização explorando uma técnica em que se utiliza um analisador girante e um ASF de fase dupla (dual phase lock-in) [7].

Usualmente, o efeito Faraday é bastante explorado em meios sólidos como cristais e vidros, dos quais alguns, apresentam altos valores para a constante de Verdet. No entanto, o estudo de líquidos é interessante porque estes apresentam algumas vantagens com relação aos sólidos, como a facilidade de preparação das amostras, bem como a possibilidade de estudar uma grande variedade de materiais. Como sabemos, amostras sólidas necessitam de preparos de lapidação e polimento que impõem dificuldades na caracterização de um grande número de materiais, o que não acontece com líquidos dentro de um solenoide oco com janelas. Dessa forma, escolhemos estudar dois solventes comuns e abundantes: água e etanol e misturas controladas desses dois líquidos. Neste caso, podemos testar a sensibilidade dos dois métodos propostos com o uso de um ASF, através de medidas da dispersão, pela da constante de Verdet em função do comprimento de onda, bem como pelo monitoramento da mudança dessa constante em função da mistura dos dois solventes.

\section{Princípios Fundamentais da Amplificação Sensível à Fase}

A técnica de detecção sensível à fase é utilizada para medir sinais muito baixos mesmo que eles estejam mascarados no meio de ruídos muito grandes. Grosso modo, ela trabalha com a eliminação de ruídos espúrios a partir de uma "filtragem" muito eficiente com operações matemáticas e também com grande amplificação dos sinais de interesse [8]. Como o próprio nome da técnica indica, o ASF precisa de um sinal de referência de fase e também de frequência bem definido.

Para ilustrar o princípio de funcionamento de um ASF temos que mostrar como são as operações matemáticas fundamentais que acontecem dentro do aparelho. O ASF gera um sinal interno tipo senoidal sincronizado com uma referência (interna ou externa, conforme o caso) com uma certa fase arbitrária $\theta_{(\text {ref })}$ :

$$
V_{(\text {ref })}=V_{0(\text { ref })} \operatorname{sen}\left(\omega_{(\text {ref })} t+\theta_{(\text {ref })}\right),
$$

o sinal a ser medido deve ser modulado com a mesma frequência de referência, mas que pode ter uma outra fase $\theta_{(\text {sig })}$ qualquer:

$$
V_{(s i g)}=V_{0(s i g)} \operatorname{sen}\left(\omega_{(s i g)} t+\theta_{(s i g)}\right) .
$$

Para a detecção sensível à fase, o que o ASF faz internamente é uma multiplicação desses dois sinais:

$$
\begin{aligned}
V_{A S F}= & V_{0(r e f)} \operatorname{sen}\left(\omega_{(r e f)} t+\theta_{(r e f)}\right) \\
& * V_{0(\operatorname{sig})} \operatorname{sen}\left(\omega_{(s i g)} t+\theta_{(s i g)}\right) .
\end{aligned}
$$

E que em outra representação matemática, a Eq. (3) pode ser escrita como:

$$
\begin{aligned}
V_{A S F}= & \frac{1}{2} V_{0(\text { ref })} V_{0(\text { sig })} \cos \left(\left(\omega_{(s i g)}-\omega_{(r e f)}\right) t\right. \\
& \left.+\theta_{(\text {sig })}-\theta_{(\text {ref })}\right)-\frac{1}{2} V_{0(\text { ref })} V_{0(\text { sig })} \cos \left(\left(\omega_{(\text {sig })}\right.\right. \\
& \left.\left.+\omega_{(\text {ref })}\right) t+\theta_{(\text {sig })}+\theta_{(\text {ref })}\right)
\end{aligned}
$$

Neste caso, o primeiro e o segundo termo oscilam com a diferença e soma das frequências, respectivamente. Caso esse sinal passe por um filtro "passa baixa" (frequências), a componente de mais alta frequência (soma) é removida, restando apenas o primeiro termo da diferença de frequências. Na hipótese das frequências serem iguais, como proposto, este termo se torna uma constante:

$$
V_{A S F}=\frac{1}{2} V_{0(\text { ref })} V_{0(s i g)} \cos \left(\theta_{(s i g)}-\theta_{(r e f)}\right) .
$$

Como pode ser observado, esse sinal constante é uma função cossenoidal da diferença de fase e, portanto, o seu valor máximo ocorre quando o cosseno vale 1 ou -1 (máximo negativo). Em outras palavras, o sinal lido no ASF muda conforme se varia a fase relativa entre o Sinal e a Referência. Num ASF de fase única, essa operação de encontrar o valor máximo pode ser feita manualmente ou automaticamente no aparelho pela varredura da fase na qual se busca esse valor máximo. Normalmente, é mais fácil encontrar a fase em que o valor é nulo (ou zero) e, posteriormente, fazer uma mudança de fase de $90^{\circ}$, onde sabe-se que o valor é máximo, positivo ou negativo.

É possível eliminar essa dependência do sinal com a fase, para isso, pode-se utilizar um segundo ASF ajustado para ler o sinal "fora de fase" ou em quadratura (90 graus fora de fase), ou seja, utilizando um sinal de referência "senoidal", $V_{(\text {ref })}=V_{0(\text { ref })} \operatorname{sen}\left(\omega_{(\text {ref })} t+\right.$ $\left.\theta_{\left(\text {ref }+90^{\circ}\right)}\right)$, e, dessa forma, o sinal constante no ASF vai depender do seno da diferença de fase:

$$
\left(V_{A S F}\right)_{a n t i}=\frac{1}{2} V_{0(r e f)} V_{0(s i g)} \operatorname{sen}\left(\theta_{(s i g)}-\theta_{(r e f)}\right) .
$$

Em resumo, pode-se ter dois sinais contínuos, um que é proporcional ao cosseno e outro ao seno, que são chamados usualmente de componentes $X$ e $Y$ (em fase e em quadratura), respectivamente. Desta forma, a magnitude $R$ do sinal pode ser obtida independentemente da fase relativa entre o sinal e a referência, pois:

$$
R=\sqrt{\left(X^{2}+Y^{2}\right)} .
$$

Como comentamos, pode-se medir $R$ utilizando-se dois ASF distintos de fase única ou um único ASF de fase 
dupla. Os ASF de fase dupla possuem dois sinais de referência internos, um "senoidal" (em fase) e outro "cossenoidal" (em quadratura).

Os ASF de fase dupla também permitem medidas de fases (ou ângulos), $\phi=\theta_{(s i g)}-\theta_{(r e f)}$, com precisão pois:

$$
\emptyset=\operatorname{atan}\left(\frac{Y}{X}\right)
$$

de forma que mesmo que a diferença de fase mude com o tempo (drift), ela poderá ser medida com grande precisão.

É possível entender que uma das características mais importantes do uso dessa técnica sensível à fase é a possibilidade da eliminação de ruídos espúrios, uma vez que os ruídos normalmente têm frequência e fase aleatórias, cujos valores médios são nulos. Dada a excelente filtragem de ruídos obtidos com as operações matemáticas, pode-se trabalhar com grandes amplificações dos sinais elétricos a serem lidos. Dessa forma se justifica o nome amplificação sensível à fase e permite que o ASF possa medir voltagens muito pequenas ( pico volts).

Outro assunto que costuma gerar confusão é a leitura do valor fornecido pelo ASF. Normalmente o ASF fornece o valor em volts rms (root mean square), ou seja, a indicação de um sinal de $1 \mathrm{~V}(\mathrm{rms})$, corresponde à leitura de um sinal cuja componente senoidal tem uma amplitude de $1 \mathrm{~V}(\mathrm{rms})$, ou $\sim 2,8 \mathrm{~V}(\mathrm{pp})$ (pico-a-pico) na frequência do sinal de referência. Portanto, caso o sinal de entrada tenha forma senoidal a leitura é direta, no entanto, para outro tipo de sinal (onda quadrada, dente de serra, triangular, etc.), o ASF detecta a primeira componente senoidal de Fourier do sinal. Por exemplo, uma onda quadrada na entrada do ASF com $2 \mathrm{~V}(p p)$ resulta numa leitura de $1,273 / \sqrt{ } 2 \mathrm{~V}$ ( rms) ( 0,9 V), pois a primeira componente de Fourier de uma onda quadrada é $1,273 \operatorname{sen}(\omega t)$.

\section{Conceitos Básicos do Efeito Faraday}

Como já mencionamos, o efeito Faraday é a rotação do plano de polarização da luz linearmente polarizada durante a propagação por um meio sujeito a um campo magnético externo paralelo à direção de propagação. Em outras palavras, é um efeito oriundo da birrefringência circular induzida pelo campo magnético no meio. $\mathrm{O}$ efeito Faraday, por sua vez, depende da contante de Verdet, que por definição, é dada pela seguinte equação:

$$
V=\frac{\theta}{B L}=\frac{e}{2 m c} \lambda \frac{d n}{d \lambda},
$$

em que, $\theta$ é o ângulo que o plano de polarização gira, $B$ é o valor do módulo do campo magnético, $L$ o comprimento do meio, e é a carga do elétron (negativa), $m$ a massa do elétron, $c$ a velocidade da luz no vácuo, $\lambda$ o comprimento de onda e $d n / d \lambda$ é a derivada do índice de refração do meio com relação ao comprimento de onda.
Uma forma de se medir a constante de Verdet usando um ASF é com a aplicação de campos magnéticos alternados fracos (Fig. 1). Neste caso, uma luz monocromática linearmente polarizada, ao passar por um meio sujeito a um campo magnético alternado, sofrerá uma rotação também alternada. Para medir a magnitude dessa rotação, coloca-se um analisador estático com o eixo da polarização à $45^{\circ}$ com relação à polarização de entrada. Dessa forma, um detector acoplado ao ASF capta a máxima variação da intensidade da luz transmitida pelo analisador [6]. Nesta configuração, é possível medir o ângulo de rotação médio da polarização por:

$$
\theta=\frac{1}{2} \frac{\Delta V_{0}}{V_{0}}
$$

em que, $\Delta V_{0}$ é a variação (modulação) do sinal medido pelo ASF e é proporcional à intensidade média da luz que passa pelo analisador na presença do campo magnético alternado e $V_{0}$ corresponde ao sinal de luz constante que passa pelo analisador com um ângulo de $45^{\circ}$. É importante salientar que o ângulo de $45^{\circ}$ do analisador é importante pois afeta tanto $\Delta V_{0}$ quanto $V_{0}$ e, consequentemente, a medida do ângulo $\theta$. Além disso, é neste ângulo que a variação da intensidade de luz transmitida é linear com o campo magnético.

No caso de líquidos, uma configuração simples consiste em construir um solenoide oco que possa envolver todo o meio óptico. Neste caso, por simplicidade, o comprimento da bobina é o mesmo do meio. Além disso, para minimizar os efeitos de borda no campo magnético, utiliza-se um solenoide muito mais longo que o seu diâmetro. Neste caso, o valor teórico do módulo do campo magnético de um solenoide pode ser encontrado pela equação:

$$
B=\frac{\mu_{0} N A}{L}
$$

em que $\mu_{0}$ é a permeabilidade magnética do vácuo $(4 \pi \times$ $10^{-7}$ T.m/A ou $4 \pi \times 10^{-1}$ G.cm/A), $N$ é o número de espiras, $A$ é a corrente e $L$ é o comprimento da bobina.

Existem algumas variáveis importantes para a realização do experimento. Uma delas é a frequência do campo magnético, não há nenhuma restrição quanto à frequência de oscilação do campo magnético, mas ela deve ser escolhida de acordo com o tempo de resposta do detector e a impedância do circuito utilizado. Outra, é a razão dada na Eq. 10 que é independente da intensidade da luz, mas tem que se ter o cuidado de evitar a saturação do detector

Combinando as equações $(9), 10)$ e 11 temos:

$$
V=\frac{\Delta V_{0}}{2 V_{0}} \frac{1}{\mu_{0} N A}
$$

É possível estabelecer que a constante de Verdet pode ser obtida pelo coeficiente angular de um gráfico em 
que os eixos $x$ e $y$ são, respectivamente, os valores das correntes induzidas na bobina $(A)$ e a variação da luz polarizada $\left(\Delta V_{0}\right)$ que cresce linearmente com o aumento da corrente. Portanto:

$$
V=\frac{C_{a}}{2 V_{0} \mu_{0} N}
$$

em que $c_{a}$ é o coeficiente angular da reta.

Já para o caso de aplicações de campos magnéticos estáticos, para se medir pequenos ângulos de rotação da polarização, é possível utilizar técnicas sensíveis à fase com o uso de dois ASF ou um ASF de fase dupla. Como vimos na seção 2, em que os princípios fundamentais de um amplificador sensível à fase são expostos, é possível medir com grande precisão atraso de fases (ângulos). Neste caso, como o campo magnético é estático, para o funcionamento das técnicas sensíveis à fase em que é necessário sinais oscilantes, é preciso utilizar um polarizador girante para modular o feixe de luz.

É possível determinar com precisão pequenos ângulos de rotação da polarização a partir da medida de atraso de fase num ASF de fase dupla. Para isso é necessário introduzir um polarizador (analisador) girante [7] e que pode ser feito por um dispositivo adequado com motor. Nesta técnica, o ângulo medido pelo ASF é o dobro do real e, portanto, isso deve ser levado em conta na aplicação direta da Eq. (9) para se determinar a constante de Verdet.

\section{Experimento}

As Figs. 1 2 e 3 mostram esquematicamente como são as montagens experimentais em que são usados campos magnéticos alternados e contínuos. Basicamente o sistema experimental possui um solenoide, uma fonte de luz, fontes de tensão AC ou DC, filmes polarizadores, um fotodetector de luz e um ASF comercial. Para ser mais específico, por exemplo, no caso da montagem

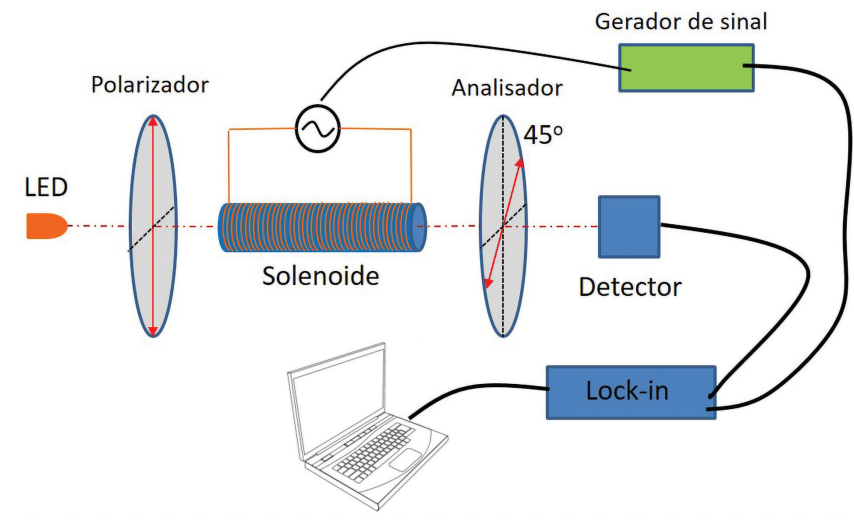

Figura 1: Diagrama esquemático do aparato experimental utilizando tensão alternada. O gerador de tensão AC (gerador de sinal) fornece como referência para o ASF (lock-in) a frequência e a fase.
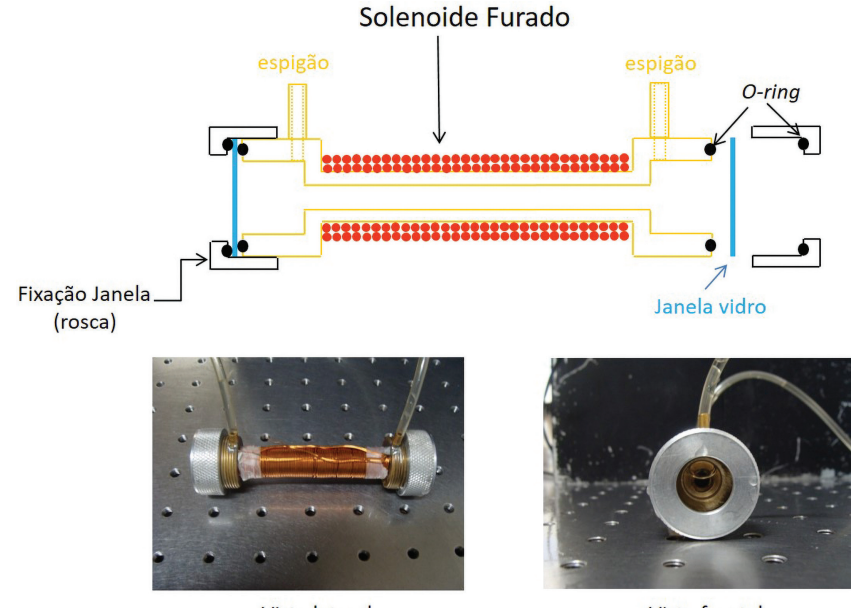

Vista frontal

Figura 2: Corte esquemático e duas fotos do solenoide utilizado no experimento. A região do solenoide com 704 espiras tem $7,4 \mathrm{~cm}$ de comprimento, um furo ao longo do solenoide de $5 \mathrm{~mm}$ de diâmetro e 2 janelas finas de vidro de $1^{\prime \prime}$ de diâmetro nas extremidades.

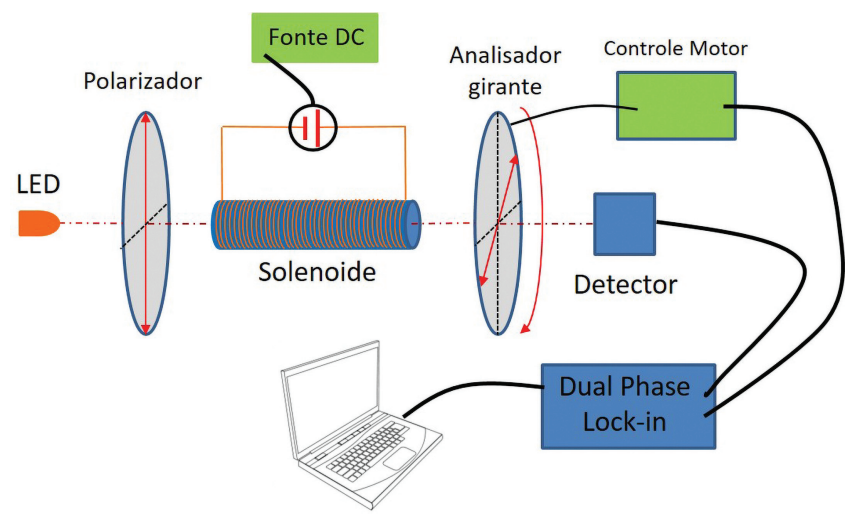

Figura 3: Diagrama esquemático do aparato experimental utilizando tensão contínua. O sistema de rotação do analisador fornece como referência para o ASF (lock-in) a frequência e a fase.

experimental usando tensão alternada, temos o LED, cuja luz não polarizada passa por um polarizador linear (filtro polaroid) fixo na entrada do solenoide furado. $\mathrm{O}$ solenoide é alimentado por uma tensão senoidal regulável tanto em amplitude quanto em frequência. Pode-se usar um gerador de sinal ou, ainda melhor, usar a própria fonte interna do ASF, que é uma fonte bastante estável e precisa. Normalmente, os ASFs comerciais possuem um gerador de sinal senoidal interno. Na saída do solenoide, é posicionado um segundo polarizador (analisador), e logo a seguir, um fotodetector de silício de área grande (DET100A, Si Biased Detector, Thorlabs) ligado numa carga resistiva e na entrada do ASF (7225 DSP Lockin Amplifier, Signal Recovery) que, por sua vez, pode ser conectado a um sistema opcional de aquisição de dados acoplado a um computador portátil. Como já foi mencionado, para maximização do sinal detectado pelo 
ASF, o ângulo do analisador é fixado em 45 graus com relação à polarização de entrada.

Como pode ser visto, para se ter um baixo custo do sistema experimental, ao invés de lasers, foram utilizados LEDs para fornecerem luzes relativamente monocromáticas. Além disso, foram empregados filmes polarizadores plásticos convencionais, um gerador de sinal simples e detectores comerciais de silício tipo PIN. O ASF é o equipamento mais caro deste experimento.

Para a passagem de luz, o corpo do solenoide em latão, tem um furo central longitudinal de $5 \mathrm{~mm}$ de diâmetro e duas janelas de vidro, de $1^{\prime \prime}$ de diâmetro e $1 \mathrm{~mm}$ de espessura, removíveis nas extremidades. Para a vedação do líquido, foram utilizados anéis de borracha (O-rings). Além disso, nas duas extremidades do solenoide, foram fixados dois espigões para introdução e troca de líquido sem a formação de bolhas e sem a necessidade de remoção do solenoide da montagem experimental. Para o solenoide foi utilizado um fio envernizado $\mathrm{N}^{\circ} 22$ de transformador com um total de 704 espiras. A Fig. 2 mostra o solenoide utilizado.

No caso de aplicação de campo magnético alternado, segundo a Eq. (10), para se determinar o ângulo de rotação médio, é necessário saber o valor de $\Delta V_{0}$ fornecido pelo ASF, e o valor de $V_{0}$, tensão DC do fotodetector, que pode ser obtida com um multímetro de precisão. Normalmente, a saída do fotodetector tem um sinal de fundo (background) que pode atrapalhar a determinação exata de $V_{0}$, portanto, deve-se tomar um cuidado especial com esse sinal.

A impedância da carga resistiva no detector tem grande influência na magnitude do sinal lido no ASF e no voltímetro. Utilizamos uma carga resistiva de $330 \mathrm{k} \Omega$ que se mostrou apropriada para uma boa magnitude de tensões $\mathrm{DC}\left(V_{0}\right)$ e da tenção $\mathrm{AC}$ no $\operatorname{ASF}\left(\Delta V_{0}\right)$ num regime de baixa frequência de modulação $(200 \mathrm{~Hz})$. Impedâncias mais altas podem ser usadas para leituras de valores maiores, no entanto, tem que se tomar cuidado com a frequência máxima em que o circuito responde sem saturação. Para o nosso circuito, com a carga resistiva utilizada, foi estimado que o limite máximo de frequência seria de $\sim 1200 \mathrm{~Hz}$, portanto, a aplicação de uma frequência de $200 \mathrm{~Hz}$ é bastante segura para evitar qualquer tipo de saturação devido ao tempo de resposta do circuito.

A configuração experimental para o caso do uso de campo magnético estático é bastante similar à anterior, com a diferença que, no lugar do gerador de sinal, é utilizada uma fonte de tensão contínua simples $(D C$ Power Supply, MPL-1305M, 30 V, 3 A, Minipa), em que é possível aplicar uma corrente muito maior que a empregada na situação de campo magnético alternado. O limite da fonte de tensão utilizada é de $3 \mathrm{~A}$, mas, para não aquecer muito o solenoide e nem sobrecarregar a fonte de tensão, a corrente foi limitada em 2 A. Neste caso, para se determinar o ângulo estático de rotação da polarização usando o ASF, pode-se usar uma técnica alternativa de polarizador girante e um ASF de fase dupla 7].

Com relação ao analisador girante, foi feito um sistema mecânico capaz de girar um polarizador linear com motor elétrico e ao mesmo tempo fornecer um sinal de referência (trigger), Fig. 4. Como um polarizador linear é degenerado a cada $180^{\circ}$, a frequência de referência do analisador é $2 \omega$, em que $\omega$ é a frequência de rotação do analisador.

É fácil perceber que o analisador girante causa uma modulação tipo cosseno ao quadrado do sinal de luz no detector (lei de Malus) (Fig. 5). Caso não haja campo magnético estático aplicado, a polarização de entrada é mantida à medida que a luz se propaga dentro do meio no solenoide. Nesta situação, pode-se considerar que há um atraso da fase nulo $(\Delta \phi=0)$. Na medida em que um campo magnético é aplicado e a polarização linear de saída do meio muda de direção, o ASF é capaz de detectar uma mudança de fase (ângulo) que é justamente

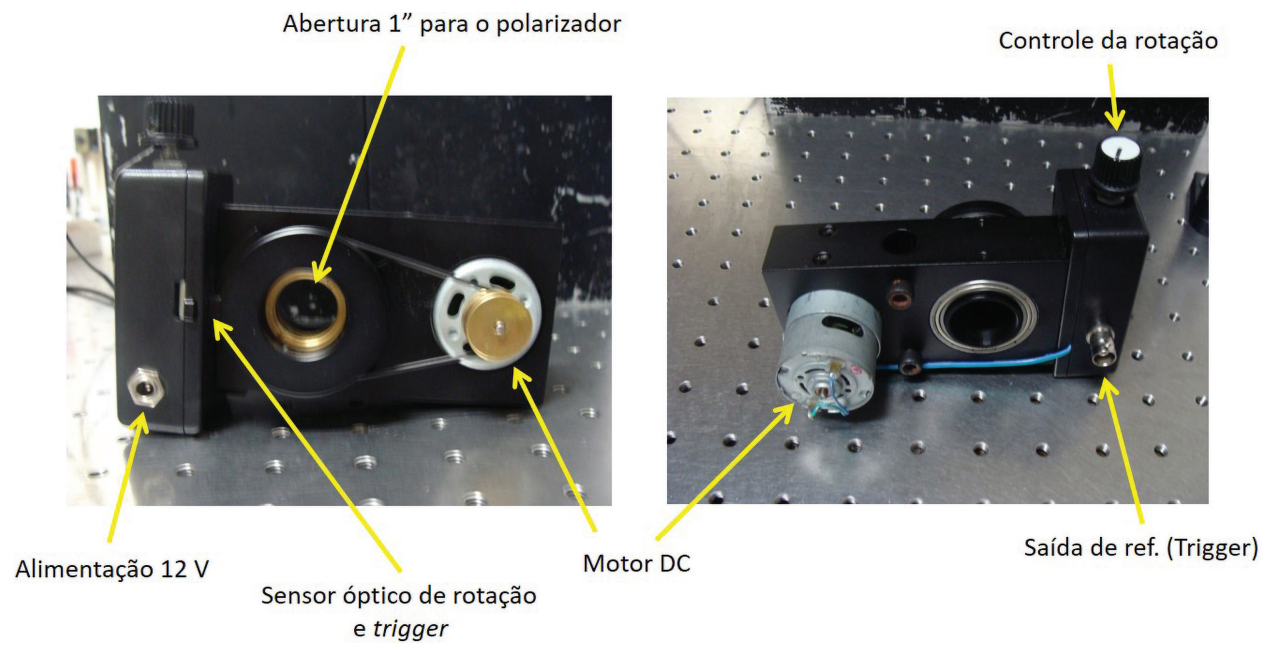

Figura 4: Fotos do sistema de rotação do polarizador (analisador). 


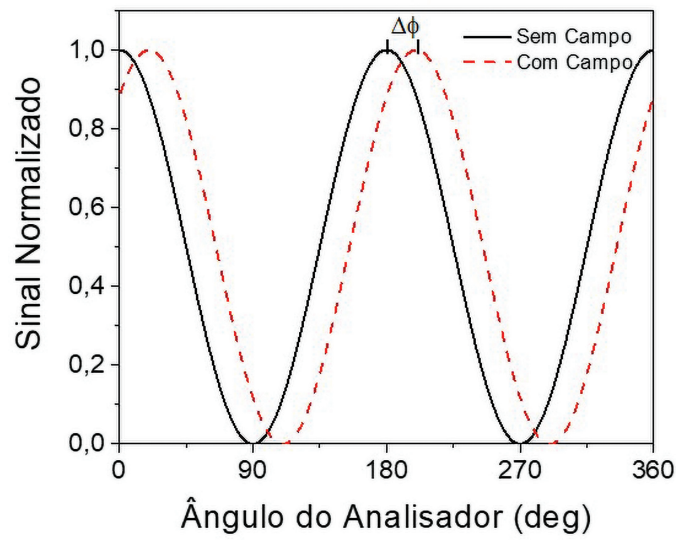

Figura 5: Diagrama esquemático do sinal de luz no detector com e sem campo magnético aplicado. O ASF de duas fases é capaz de medir a mudança de fase $\Delta \phi$ adquirida com a aplicação do campo magnético.

o dobro do ângulo de rotação da polarização devido à lei de Malus. Mesmo que essa rotação seja pequena, o ASF de duas fases pode medir esse ângulo com precisão.

Neste caso, o ângulo de rotação é determinado pelo ASF, ou seja, é uma medida mais direta que no caso anterior em que se utilizam campos magnéticos alternados, pois, sabendo-se o ângulo de rotação, pela Eq. (9) pode se determinar diretamente a constante de Verdet.

\section{Resultados e Discussões}

As medidas foram feitas na água pura, no etanol puro e nas misturas percentuais entre a água e o etanol numa variação de $10 \%$ em volume entre uma mistura e outra. Para observar a influência da dispersão foram usados três LEDs de cores diferentes: azul (480 nm), verde (540 $\mathrm{nm})$ e vermelho $(640 \mathrm{~nm})$. Apesar de não serem tão monocromáticos como os lasers, eles são muito mais simples, de baixo custo e adequados para mostrar a dispersão da constante de Verdet.

Usando o campo alternado foram realizadas medidas sistemáticas nos diferentes líquidos usando diferentes correntes no solenoide para os três LEDs. Para cada medida foi feito um gráfico de sinal lido no ASF v.s. corrente aplicada, Fig. 6. O limite da fonte de tensão do ASF (7225 DSP) é de $5 \mathrm{~V}$ e, neste caso, a corrente máxima foi em torno de $65 \mathrm{~mA}$. Pelo coeficiente angular $c_{a}$ de cada gráfico é possível obter a constante de Verdet com maior precisão (Eq. (13)). Há uma incerteza na leitura da corrente e da voltagem devido a precisão dos aparelhos utilizados, mas isso não foi levado em conta pois havia um erro estatístico maior. A barra de erro das medidas $( \pm 0,2 \mathrm{rad} /($ T.m $))$ foi estimada a partir da variação típica observada para a constante de Verdet entre medidas distintas realizadas independentemente. O resumo dos resultados para a constante de Verdet,

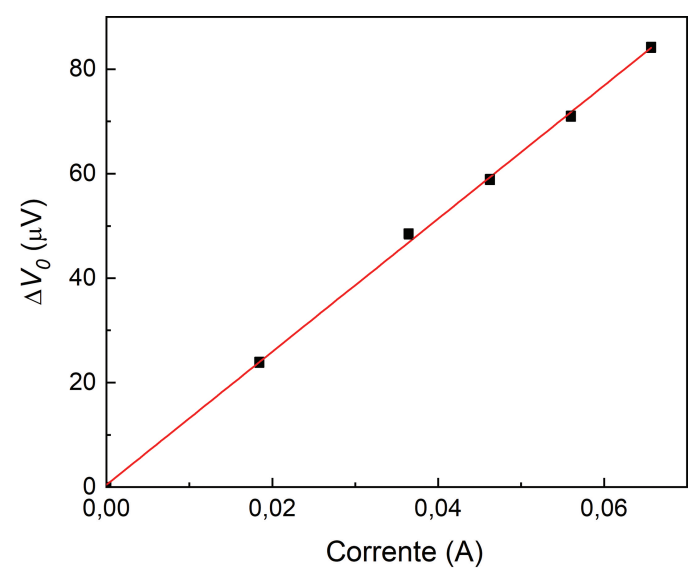

Figura 6: Exemplo de uma típica medida do efeito Faraday usando campo $A C$ em gráfico de $\Delta V_{0}$ lido no ASF em função da corrente aplicada no solenoide. Pelo ajuste linear nas medidas experimentais se obtém o coeficiente angular $\left(c_{a}=0,00126\right)$ para a determinação da constante de Verdet. Nestas medidas utilizou-se amostra com $30 \%$ água, voltagem no fotodetector $V_{0}=0,207 \mathrm{~V}$, LED verde e frequência de oscilação de $200 \mathrm{~Hz}$.

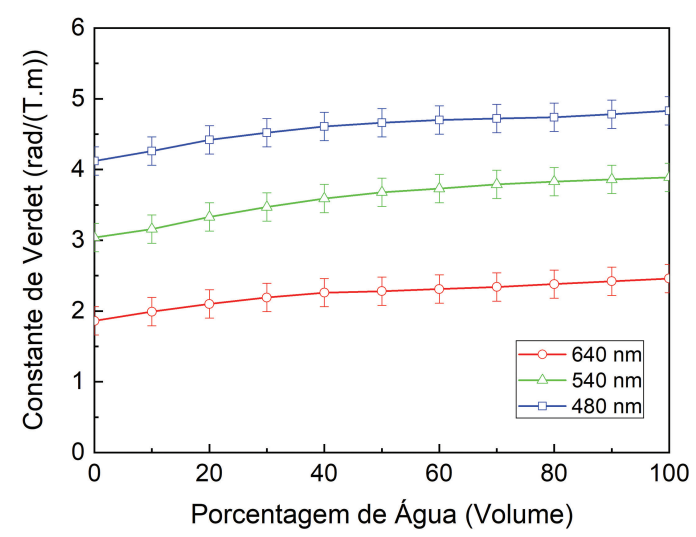

Figura 7: Constante de Verdet em função da concentração de água no etanol, para três comprimentos de onda, obtida com o campo magnético alternado. A linha contínua é apenas para ajudar a guiar os olhos.

para todas as concentrações estudadas e para os três comprimentos de onda, é apresentado na Fig. 7.

Com a aplicação de campo magnético estático, a medida é mais intuitiva, pois é possível obter diretamente o ângulo de rotação da polarização no ASF. Neste caso, é necessário aplicar um campo magnético contínuo maior para que o ângulo de rotação seja mensurável com precisão. Para cada líquido foram feitas pelo menos 5 medidas da rotação na corrente máxima escolhida $(2 \mathrm{~A})$. Todos os resultados obtidos para as diferentes amostras e diferentes comprimentos de onda foram compilados e são mostrados na Fig. 8

Os valores obtidos com corrente contínua foram ligeiramente maiores que com corrente alternada. Como pode ser observado (Figs. 7 e 8), as constantes de Verdet do etanol foram sempre menores que as da 


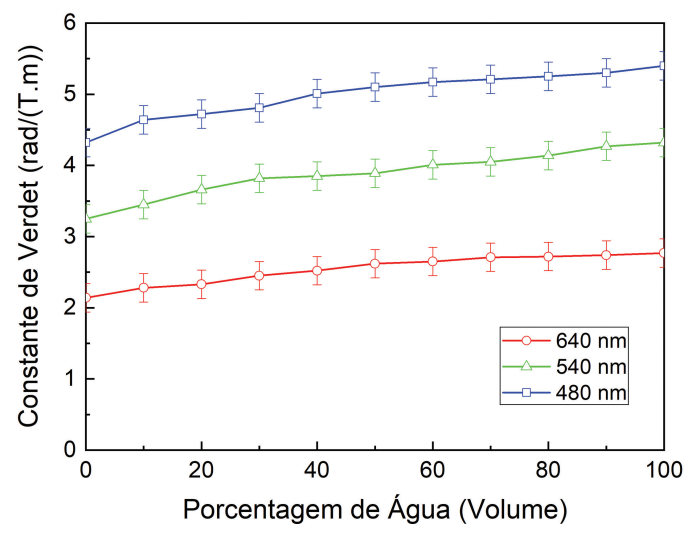

Figura 8: Constante de Verdet em função da concentração de água no etanol, para três comprimentos de onda, obtida com o campo magnético estático. A linha contínua é apenas para ajudar a guiar os olhos.

água pura nos três comprimentos de onda. A mistura entre os dois solventes leva a valores intermediários proporcionais à concentração de um dos solventes. É possível observar que a curva da constante de Verdet não segue uma equação linear em função da concentração em volume da água. Como sabemos, o valor da constante de Verdet está relacionada à dispersão do índice de refração do meio (Eq. (9)). Dessa forma, é possível inferir que provavelmente a variação do índice de refração em função da concentração de um dos solventes deva ser similar às curvas obtidas em nossas medidas. Ademais, é fácil notar a dispersão como função do comprimento de onda das constantes de Verdet para todos os líquidos caracterizados usando esses três comprimentos de onda.

Como os solventes puros possuem equações de índices de refração conhecidas é possível fazer um cálculo da constante de Verdet. Usando as equações de dispersão da água e do etanol, é possível determinar a constante de Verdet teórica pela Eq. (9). Usamos como equação de dispersão da água [9]

$$
n^{2}-1=\frac{0,75831 \lambda^{2}}{\lambda^{2}-0,01007}+\frac{0,08495 \lambda^{2}}{\lambda^{2}-8,91377}
$$

e do etanol [10]:

$$
n^{2}-1=\frac{0,83189 \lambda^{2}}{\lambda^{2}-0,00930}-\frac{0,15582 \lambda^{2}}{\lambda^{2}+49,45200}
$$

A derivada do índice de refração $(d n / d \lambda)$ pode ser feita numericamente ou diretamente pelas Eqs. (14) e (15). As curvas das constantes de Verdet obtidas são mostradas na Fig. 9. bem como os resultados experimentais para os solventes puros, obtidos com as duas técnicas propostas.

Como pode ser observado, os resultados obtidos pelos dois métodos experimentais aqui propostos para medida das constantes de Verdet para os solventes puros, compilados nas Tabelas 1 e 2 foram todos um pouco menores que os reportados na Ref. 6]. Esses valores menores podem ser entendidos se considerarmos que o

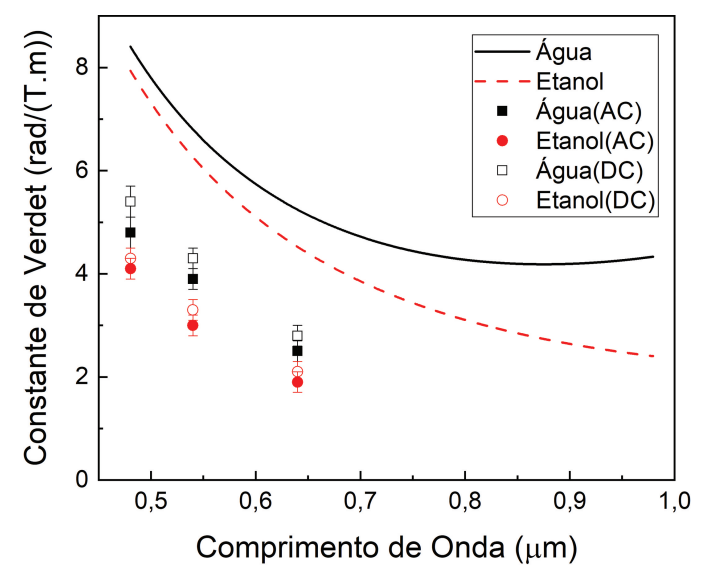

Figura 9: Resultados obtidos experimentalmente para água e etanol com campo AC e DC. Curvas das constantes de Verdet da água e do etanol obtidas a partir da equação de dispersão dos índices de refração.

Tabela 1: Constante de Verdet obtida para a água a partir dos dados experimentais usando ASF com campo magnético alternado, $V_{\mathrm{AC}}$, e contínuo, $V_{\mathrm{DC}}$, além de valores obtidos

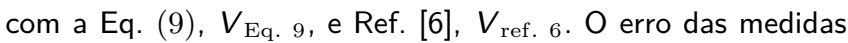
experimentais é de $\pm 0,2 \mathrm{rad} /$ (T.m).

\begin{tabular}{lcccc}
\hline $\begin{array}{c}V_{\text {AC }} \\
\lambda(\mathrm{nm})\end{array}$ & $\begin{array}{c}V_{\mathrm{DC}} \\
(\mathrm{rad} /(\mathrm{T} . \mathrm{m}))\end{array}$ & $\begin{array}{c}V_{\text {Eq. }} \\
(\mathrm{rad} /(\mathrm{T} . \mathrm{m}))\end{array}$ & $\begin{array}{c}V_{\text {ref. }} \\
(\mathrm{rad} /(\mathrm{T} . \mathrm{m}))\end{array}$ & $(\mathrm{rad} /(\mathrm{T} . \mathrm{m}))$ \\
\hline 480 & 4,8 & 5,4 & 8,4 & 5,8 \\
540 & 3,9 & 4,3 & 6,8 & 4,4 \\
640 & 2,5 & 2,8 & 5,2 & 3,3 \\
\hline
\end{tabular}

Tabela 2: Constante de Verdet obtida para o etanol a partir dos dados experimentais usando ASF com campo magnético alternado, $V_{\mathrm{AC}}$, e contínuo, $V_{\mathrm{DC}}$, além de valores obtidos

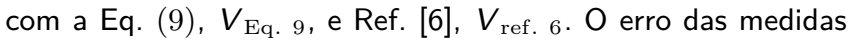

\begin{tabular}{|c|c|c|c|c|}
\hline$\lambda(\mathrm{nm})$ & $\begin{array}{c}V_{\mathrm{AC}} \\
(\mathrm{rad} /(\mathrm{T} . \mathrm{m}))\end{array}$ & $\begin{array}{c}V_{\mathrm{DC}} \\
(\mathrm{rad} /(\mathrm{T} . \mathrm{m}))\end{array}$ & $\begin{array}{c}V_{\text {Eq. } 9} \\
(\operatorname{rad} /(\text { T.m) })\end{array}$ & $\begin{array}{c}V_{\text {ref. } 6} \\
(\mathrm{rad} /(\mathrm{T} . \mathrm{m}))\end{array}$ \\
\hline 480 & 4,1 & 4,3 & 7,9 & 4,9 \\
\hline 540 & 3,0 & 3,3 & 6,3 & 3,7 \\
\hline 640 & 1,9 & 2,1 & 4,5 & 2,6 \\
\hline
\end{tabular}
experimentais é de $\pm 0,2 \mathrm{rad} /($ T.m) .

campo magnético real do solenoide deva ser menor que o calculado teoricamente para o caso de um solenoide ideal, Eq. (11), devido aos efeitos de bordas.

Em termos comparativos, levando em conta as barras de erro, apenas os valores obtidos para a água com os dois métodos para o LED azul não foram compatíveis. Para todas as demais medidas os valores obtidos pelas duas técnicas são próximos. Acreditamos que essa discordância no LED azul se deva ao uso de filmes polaroides convencionais pois eles polarizam com menos eficiência a cor azul do espectro visível, levando a uma incerteza maior para as medidas realizadas com campo magnético alternado. Como já mencionamos, o método em que se usa campos magnéticos alternados é bastante 
dependente da qualidade da polarização da luz para prover os corretos valores de $\Delta V_{0}$, e $V_{0}$ da Eq. (10), e provavelmente os valores obtidos com o LED azul com tensão AC estão subestimados com relação aos obtidos com tensão DC. A técnica de medida com campo DC é pouco afetada pela qualidade da polarização da luz uma vez que se mede apenas a direção da polarização.

Pelos resultados obtidos é possível destacar algumas particularidades dos dois métodos para se medir a constante de Verdet. Em termos de precisão, a técnica com campo contínuo demonstrou ser um pouco mais interessante pois ela levou à obtenção de valores mais próximos com relação aos da literatura [6]. Por outro lado, a técnica com tensão AC é muito mais simples de ser implementada por utilizar apenas equipamentos comerciais, o que não é o caso de aplicação de tensão DC, pois neste caso é necessário desenvolver um sistema motorizado de rotação do analisador. Outras observações importantes são, que a técnica $\mathrm{AC}$ também usa menores correntes que a com tensão DC, a medida com campo DC é mais intuitiva por medir diretamente ângulos (fase) e permitir a determinação do sentido de rotação da polarização, que é muito importante para saber o sinal da constante de Verdet, por exemplo.

Também fizemos uma análise em que podemos comparar com os valores obtidos pelas equações dos índices de refração (Eq. (9)). Neste caso, houve uma maior discrepância entre os valores obtidos experimentalmente e os calculados pela Eq. (9) ( $2 \times$ maior), entretanto, isso já era esperado uma vez que os valores das constantes de Verdet, obtidos pelas dispersões dos índices de refração, normalmente não resultam em valores corretos devido à simplicidade do modelo teórico [11, [12. No entanto, esse modelo simplificado é interessante por mostrar corretas tendências e ordens de grandeza das constantes de Verdet.

Finalmente, um dos objetivos principais deste trabalho foi mostrar que, mesmo com uma montagem simples na aproximação de um solenoide ideal sem uso de amostra de calibração, é possível obter bons valores da constante de Verdet. Obviamente, resultados melhores podem ser obtidos simplesmente utilizando um solenoide mais longo e com mais espiras, bem como realizando a calibração do campo magnético efetivo com uma amostra de referência cujo valor da constante de Verdet seja bem estabelecido.

\section{Conclusões}

A utilização de ASF como também seus princípios de funcionamento foram apresentados em dois experimentos de medida das constantes de Verdet pelo efeito Faraday em líquidos. Mostramos que podem ser realizadas medidas com campos magnéticos alternados ou estáticos. Foi dada uma especial atenção para o princípio de funcionamento do ASF para que ele possa ser explorado para a obtenção de valores mais precisos de outras grandezas físicas, quando possível. Como é sabido, o ASF é um importante instrumento de pesquisa que pode ser amplamente utilizado em diferentes experimentos para medida de pequenos sinais elétricos e medida de fase com precisão. Destacamos que a vantagem do uso de um ASF está na melhor sensibilidade e na consequente aplicação de menores campos magnéticos, possibilitando a implementação de sistemas experimentais compactos e simples, adequados para laboratórios de ensino.

Realizamos medidas da constante de Verdet da água e do etanol puros e suas misturas em três diferentes comprimentos de onda utilizando duas técnicas, uma com campos magnéticos alternados e outra com campos magnéticos estáticos. Os valores obtidos pelas duas técnicas foram bastante adequados considerando a simplicidade dos experimentos. Foi possível observar claramente a dispersão da constante de Verdet usando luz LED: azul, verde e vermelha, bem como a mudança dessa constante em função da mistura dos dois solventes. Como referência, também fizemos o cálculo dos valores esperados para a água e o etanol puros utilizando as respectivas equações de dispersão dos índices de refração. Neste caso, houve uma discrepância maior uma vez que o modelo teórico simplificado não consegue explicar a complexidade da constante de Verdet real. No entanto, esses cálculos ajudam a revelar as corretas ordens de grandeza e dispersões.

\section{Agradecimentos}

Este trabalho teve apoio financeiro do CNPq e FAPESP.

\section{Referências}

[1] F.A. Jenkins e H.E. White, Fundamentals of optics (McGraw-Hill, New York, 2001), 4 ed.

[2] G.R. Fowles, Introduction to modern optics (Dover, New York, 1975), 2 ed.

[3] E. Hecht, Optics (Addison-Wesley, Reading, 1987), 2 ed.

[4] R.D. Guenther, Modern optics (John Wiley \& Sons, New York, 1990).

[5] F.L. Pedrotti e P. Bandettini, Am. J. Phys. 58, 542 (1990).

[6] A. Jain, J. Kumar, F. Zhou, L. Li e S. Tripathy, Am. J. Phys. 67, 714 (1999).

[7] M.L. Miguez, E.C. Barbano, S.C. Zilio e L. Misoguti, Opt. Express 22, 25530 (2014).

[8] http://www.thinksrs.com/downloads/PDFs/Applicatio nNotes/AboutLIAs.pdf, acessado em 26/04/2021.

[9] https://refractiveindex.info/?shelf $=$ main\&book $=\mathrm{H} 2$ O\&page=Kedenburg, acessado em 26/04/2021.

[10] http://www.refractiveindex.info/?shelf=organic\&boo $\mathrm{k}=$ ethanol\&page=Kedenburg, acessado em 26/04/2021.

[11] W. Grevendonk, J. Dauwen, P. Van den Keybus e B. Vanhuyse, J. Chem. Phys. 81, 3746 (1984).

[12] S. Suchat, P. Viriyavathana, P. Jaideaw, N. Haisirikul, W. Kerdsang, e S. Petcharavut, em Progress in Electromagnetics Research Symposium Proceedings (Suzhou, 2011). 